\title{
SARS-CoV-2 infection in a pediatrics STAT1 GOF patient under Ruxolitinib therapy-a matter of balance?
}

\author{
Paloma Guisado Hernández ${ }^{1}$ Pilar Blanco Lobo ${ }^{1}$ Isabel Villaoslada ${ }^{1}$ - Beatriz de Felipe ${ }^{1}$. José M. Lucena ${ }^{2}$. \\ Guillermo Martín Gutierrez ${ }^{3}$. María José Castro ${ }^{4}$ - Alicia Gutiérrez Valencia ${ }^{3}$ - María Isabel Sánchez Codez ${ }^{5}$. \\ Mirella Gaboli ${ }^{6}$. Olaf Neth ${ }^{1}$. $\cdot$ Peter Olbrich ${ }^{1}$
}

Received: 16 April 2021 / Accepted: 31 May 2021 / Published online: 9 June 2021

(c) The Author(s), under exclusive licence to Springer Science+Business Media, LLC, part of Springer Nature 2021

To the Editor,

Recently, 94 inborn errors of immunity (IEI) patients suffering from COVID-19 have been described, overall demonstrating a mild phenotype [1] although more severe disease manifestations have been suggested for patients with alterations in the interferon (IFN) signaling pathway, including auto-antibodies against type I IFN [2]. Patients with STAT1 GOF mutations show a complex and often severe phenotype, combining an increased susceptibility of fungal, (myco-) bacterial and viral infections as well as autoimmune and autoinflammatory manifestations [3]. Characteristically, in response to type I and type II IFN stimulation, these patients show STAT1 hyperphosphorylation $[3,4]$. Whether in the

Paloma Guisado Hernández, Pilar Blanco Lobo, Olaf Neth and Peter Olbrich contributed equally to this work.

Olaf Neth

oneth-ibis@us.es

1 Pediatric Infectious Diseases, Rheumatology and Immunology Unit, Hospital Universitario Virgen del Rocío, Instituto de Biomedicina de Sevilla, IBiS/ Universidad de Sevilla/CSIC, Red de Investigación Traslacional en Infectología Pediátrica RITIP, Seville, Spain

2 Immunology Unit, University Hospital Virgen del Rocío, Seville, Spain

3 Unidad de Gestión Clínica de Enfermedades Infecciosas, Microbiología Y Medicina Preventiva, Hospital Universitario Virgen del Rocío/CSIC/Instituto de Biomedicina de Sevilla (IBiS), Seville, Spain

4 Flow Cytometry, Institute of Biomedicine of Seville (IBIS), Seville, Spain

5 Pediatric Infectious Diseases, Hospital Universitario Puerta del Mar, Cádiz, Spain

6 Pediatric Respiratory Unit, Hospital Universitario Virgen del Rocío, Instituto de Biomedicina de Sevilla, IBiS/ Universidad de Sevilla/CSIC, Seville, Spain context of SARS-CoV-2 infection, the hyperactivation of the IFN-JAK1/2-STAT1 pathway would be protective (antiviral effect) or deleterious (hyperinflammation) is unclear. Ruxolitinib (a selective JAK1/2 inhibitor) has been successfully used in STAT1 GOF patients controlling many disease manifestations [5] and also resulted in improved pulmonary function and faster recovery from lymphopenia in previously healthy individuals suffering from severe COVID-19 [6].

We provide clinical and laboratory data of a STAT1 GOF adolescent suffering from mild and overall uncomplicated SARS-CoV-2 while receiving ruxolitinib treatment.

\section{Results}

\section{Clinical Case Report}

An 11-year-old girl presented in early childhood with recurrent respiratory tract infections, aphthous stomatitis, oral thrush, enteropathy, and subarachnoideal hemorrhage after rupture of a cerebral aneurysm (Supplementary Fig. 1). She was diagnosed with a previously described de novo STAT1 GOF mutation (c.1053G > T/p.Leu351Phe); initial management was supportive, consisting in antibiotic and antifungal prophylaxis. Ruxolitinib therapy was initiated in March 2018 at $0.2 \mathrm{mg} / \mathrm{kg} /$ day and progressively increased to $0.5 \mathrm{mg} / \mathrm{kg} /$ day achieving appropriate disease control. Under therapy, total STAT1 expression remained elevated, while STAT1 hyperphosphorylation and previously increased STAT1 downstream targets such as CXCL10 and PD-L1 were controlled (Fig. 1A-C). Monthly immunoglobulin infusions were started in March 2019 due to low memory B cells, diminished pneumococcal vaccine responses, recurrent respiratory tract infections, and bronchiectasis despite antimicrobial prophylaxis. SARS-CoV-2 infection from nasopharyngeal aspirate was diagnosed in October 2020 
A)

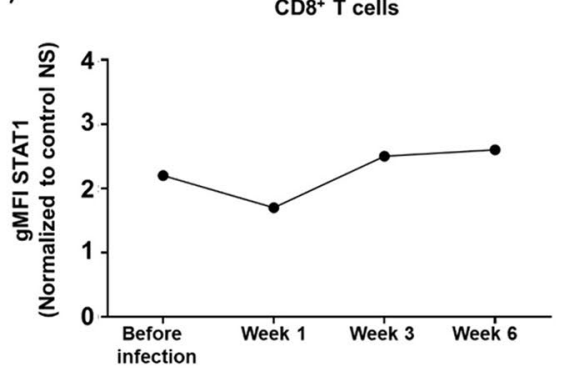

C)

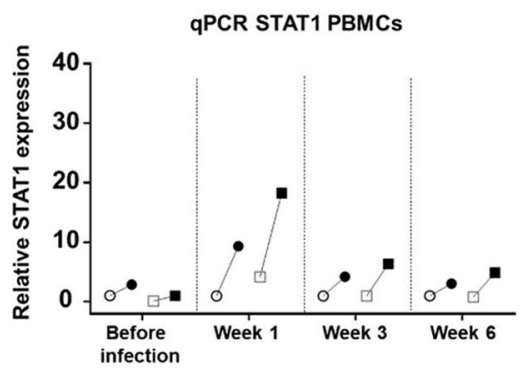

B)

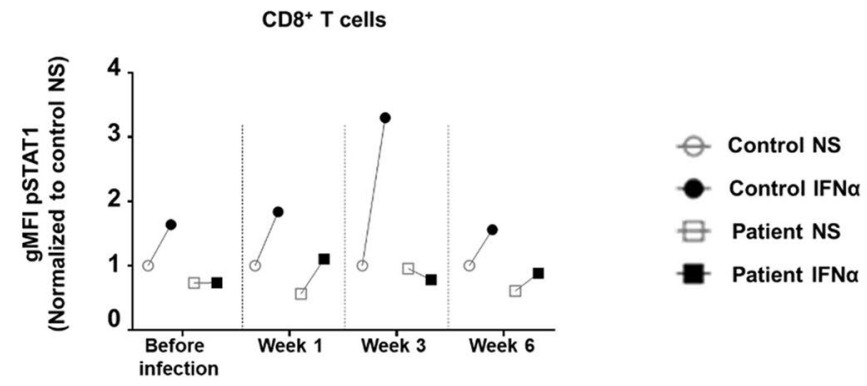

D)
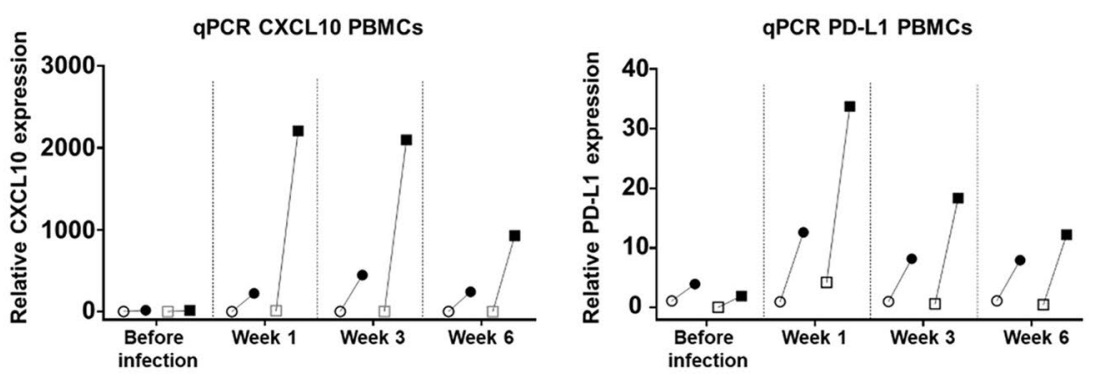

\begin{tabular}{|c|c|c|c|c|c|}
\hline \multicolumn{6}{|c|}{ SARS-CoV-2 } \\
\hline \multirow{3}{*}{ qPCR } & & Week 1 & Week 3 & Week 5 & Week 6 \\
\hline & $\begin{array}{c}\text { ORF1ab } \\
\text { (CT <35 positive) }\end{array}$ & 15.5 & 18.7 & 31.1 & 38.6 \\
\hline & $\begin{array}{c}\text { N gene } \\
(\mathrm{CT}<35 \text { positive) }\end{array}$ & 17.6 & 19.6 & 24.6 & 32.5 \\
\hline \multirow{2}{*}{ Serology } & $\underset{\text { (cut-off } \geq 1.10)}{\lg M}$ & 0.1 & 2 & 1.6 & 0.9 \\
\hline & $\underset{\text { (cut-off } \geq 15.0 \text { ) }}{\lg G}$ & $<3.8$ & 34.6 & 26 & 34.6 \\
\hline
\end{tabular}

E)

\begin{tabular}{cccc}
\hline & ELISA & & \\
\hline & Week 1 & Week 3 & Week 6 \\
\cline { 2 - 4 } IL6 & 13.6 & 2.6 & 12.6 \\
\hline $\begin{array}{c}\text { (normal values } \mathbf{0 . 5 - 3 . 9 ~} \mathrm{pg} / \mathrm{mL} \text { ) } \\
\text { IFNy } \\
\text { (normal values }<7.5 \mathrm{pg} / \mathrm{mL} \text { ) }\end{array}$ & 60.2 & 49.7 & 47.5 \\
\hline $\begin{array}{c}\text { CXCL10 } \\
\text { (normal values 51-235 } \mathrm{pg} / \mathrm{mL} \text { ) }\end{array}$ & 2819.3 & 479.7 & 543.4 \\
\hline
\end{tabular}

Fig. 1 SARS-CoV-2 infection and impact of STAT1 pathway in the presence of ruxolitinib. Evaluation of the JAK/STAT1 pathway by flow cytometry $(\mathbf{A}, \mathbf{B})$ and qPCR $(\mathbf{C})$. Non-stimulated STAT1 levels in $\mathrm{CD}^{+} \mathrm{T}$ cells before and during SARS-CoV-2 infection (A); pSTAT1 levels in CD8 + T cells pre- and post-stimulation with IFN $\alpha$ before and during SARS-CoV-2 infection (B). Expression of STAT1, CXCL10, and PD-L1 (from left to right) in PBMCs pre- and poststimulation with IFN $\alpha$ before and during SARS-CoV-2 infection (C). Data from non-stimulated and stimulated samples represented as white and black symbols, respectively. Control and patient data represented as circles and squares, respectively. The presented data in $\mathbf{A}$ and $\mathbf{B}$ has been normalized to non-stimulated samples from healthy controls. PBMCs, peripheral blood mononuclear cells; NS, non-stimulated; $I F N \alpha$, stimulated with IFN $\alpha$; $g M F I$, geometric mean fluorescence intensity. Molecular/serological SARS-CoV-2 results during infection (D). $C T$, cycle threshold; ORF1ab, Open Reading Frame ab gene. ELISA determinations of IL-6, IFN $\gamma$, and CXCL10 serum levels during infection $(\mathbf{E})$ 
by qPCR, her father being the index case. She developed only mild productive cough, low-grade fever, myalgia, and fatigue. After discussion with the family and medical team, it was decided to maintain ruxolitinib therapy. Due to fever persistence, she was admitted for observation, and empiric oral antibiotic therapy (amoxicillin-clavulanic acid) was prescribed for 7 days, while blood and sputum cultures were negative and a 20 target FILMARRAYTM Respiratory Panel (Biomerieux) confirmed SARS-CoV-2 infection only. Fever vanished at day +3 when she was discharged home. She maintained good clinical health in her follow-up visits at weeks 3 and 5; however, she developed candida vulvovaginitis at week 6 .

\section{Basic Laboratory Results}

There was overall lymphopenia with normal $\mathrm{T}$ and $\mathrm{B}$ cell distribution at week 1 , which subsequently recovered (Supplementary Table 1). Ferritin and platelets were normal and C-reactive protein (CRP) only mildly raised at week 1 , suggesting no evidence of hyperinflammation.

\section{Molecular and Serological SARS-CoV-2 Determinations}

Consecutive molecular and serology results are summarized in Fig. 1D. While SARS-CoV-2 qPCRs were strongly positive in weeks 1 and 3 and weakly positive at week 5 and 6 of infection, SARS-CoV-2 IgM and IgG were detected at weeks 3 and 5 , the latter persisting at week 6 .

\section{STAT1 and Phosphorylated STAT1 Levels}

Using whole blood without stimulation, total STAT1 levels in $\mathrm{CD} 8^{+} \mathrm{T}$ cells from the patient before and during infection were measured using flow cytometry and compared with a healthy control (HC) (Fig. 1A). STAT1 levels were overall higher in the patient compared to the $\mathrm{HC}$; this was less evident in the first week of infection.

Phosphorylated STAT1 (pSTAT1) levels after IFN $\alpha$ stimulation were lower in patient's cells than in HC during the complete follow-up, suggesting a good immunological control under ruxolitinib treatment (Fig. 1B; Supplementary Fig. 2A and 3). An increment in pSTAT1 upon IFN $\alpha$ stimulation was observed at week 1, but not before infection. In addition, stimulation of patient's $\mathrm{CD} 14^{+}$monocytes with IFN $\gamma$ showed pSTAT1 phosphorylation similar to the HC (Supplementary Fig. 2B) further suggesting an immunomodulatory effect of ruxolitinib, without complete abolishment of the IFN-JAK-STAT axis.

\section{STAT1, CXCL10, and PD-L1 Gene Expression}

In order to assess the potential effect of infection on IFN response, the transcription levels of STAT1 and STAT1 downstream genes (CXCL10 and PD-L1) were evaluated in PBMCs before and after IFN $\alpha$ stimulation (Fig. 1C). While the expression of these genes pre-infection was low and similar to HC, gene expression after IFN $\alpha$ stimulation was overall raised during infection, being highest at week 1 and subsequently decreasing.

\section{CXCL10 Secretion}

Furthermore, secretion of CXCL10 protein in supernatant from PBMCs, before and after IFN $\alpha$ stimulation, was evaluated confirming CXCL10 upregulation found in the transcription assay (Supplementary Fig. 4).

\section{IL6, IFNy, and CXCL10 Cytokine Levels}

IL6, IFN $\gamma$, and CXCL10 serum levels were measured as indicators of inflammatory responses during SARS-CoV-2 infection (Fig. 1E). The levels of these molecules were markedly elevated at week 1 , and despite an overall decrease at week 3, only IL6 reached normal levels. Six weeks after infection, CXCL10 and IFN $\gamma$ levels continued to be raised and IL6 increased again, possibly due to an intercurrent vaginal candida infection.

For laboratory details, please refer to Supplementary Methods.

\section{Discussion}

We present, the first case of an adolescent with STAT1 GOF mutation under ruxolitinib therapy who suffered from prolonged but surprisingly mild SARS-CoV-2 infection, suggesting JAK-inhibition in this clinical setting not to be harmful. This is an important observation keeping in mind that infectious complications (fungal and herpesvirus) under JAK inhibition in STAT1 GOF patients have been described [7]. Previously, only one STAT1 GOF patient with COVID-19 was reported. In that case, SARS-CoV-2 infection was asymptomatic and the patient remained on immunoglobulin substitution only [1]. At the time of hospital admission, our patient has been receiving ruxolitinib treatment for 3 years, being associated with clinical improvement of STAT1 GOF-associated symptoms without any adverse events. Despite lack of evidence for the effect of JAK inhibition on clinical outcomes of SARSCoV-2 infection, ruxolitinib treatment was maintained during infection monitoring the activity of JAK/STAT1 pathway by flow cytometry and mRNA transcription levels. 
Although increased total STAT1 levels were documented, hyperphosphorylation of STAT1 was not observed during the complete follow-up, proposing ruxolitinib to be effective in controlling cytokine hyperresponsiveness typically found in STAT1 GOF patients, even in the context of SARS-CoV-2 infection.

Despite ruxolitinib treatment, STAT1-dependent gene transcription during infection was markedly higher in the patient compared to the $\mathrm{HC}$, suggesting an ability to respond to type I interferons, known to play an important role in antiviral immunity as recently described in SARS-CoV-2 infections [2]. No age-matched HC with SARS-CoV-2 infection were available for the experiments, thereby potentially limiting the data interpretation. In addition, serum levels of IL6, IFN $\gamma$, and CXCL10 were elevated during infection, supporting the ability of the patient to develop an inflammatory response even under ruxolitinib therapy.

Interestingly, despite receiving immunoglobulin substitution, our patient efficiently mounted $\operatorname{IgM}$ and $\operatorname{IgG}$ responses to SARS-CoV-2. SARS-CoV-2 qPCR results continued to be positive up to 6 weeks, suggesting persistent infection as reported previously [8]; although in our case, infectivity was not formally assessed.

In summary, the maintenance of ruxolitinib treatment in this STAT1 GOF patient with SARS-CoV-2 infection was safe and resulted in a balanced residual type I interferon response sufficient to control viral infection whilst avoiding an uncontrolled inflammatory response. Recently, using human lung epithelial cell lines, ruxolitinib therapy was shown to normalize interferon signature genes, induced by SARS-CoV-2 infection, without affecting NF-kB-regulated genes, further supporting our observation [9]. In addition, case reports on its beneficial effect have been described in patients with myelofibrosis and rheumatoid diseases and a recent trial evaluating baricitinib and remdesivir in COVID19 pneumonia showed promising results [10-12]. Whether JAK inhibition indeed plays a role in the management of SARS-CoV-2 infections in patients with immune dysregulation syndromes as well as previously healthy subjects remains to be established by collecting prospective data from registries.

Supplementary Information The online version contains supplementary material available at https://doi.org/10.1007/s10875-021-01081-9.

Author Contribution PGH performed the experiments and wrote the paper. PBL performed the experiments and wrote the paper. IVC performed the experiments and designed the figures. BF performed experiments and reviewed the paper. JML performed the immunophenotyping and reviewed the paper. GM performed microbiologic studies and reviewed the paper. MJC overviewed the flow cytometry experiments and reviewed the paper. AGV performed experiments and reviewed the paper. MISC, medical doctor of the patient, reviewed the paper. MG, medical doctor of the patient, reviewed the paper. ON, medical doctor of the patient, designed the research study and wrote the paper. PO, medical doctor of the patient, designed the research study and wrote the paper.

Funding This work was supported by the Instituto de Salud Carlos III, Madrid (Spain) [Sara Borrell, CD20/00124 to P.B.L, Juan Rodés JR18/00042 to P.O, FIS PI19/01471 to O.N] and the Consejería de Salud, Junta Andalucía [SA0051/2020 to O.N]. A.G-V was supported by the Instituto de Salud Carlos III, cofinanced by the European Development Regional Fund ("A way to achieve Europe"), Subprograma Miguel Servet (CP19/00159).

Availability of Data and Materials Not applicable for this section.

\section{Declarations}

Ethics Approval The case study was performed according to the ethical standards. Informed consent authorized by the institutional ethics committee was obtained.

Consent to Participate Freely given and informed consent to participate in the case report was obtained from patient parents.

Consent for Publication Patient parents have consented to the submission of the case report to the journal.

Competing Interests The authors declare no competing interests.

\section{References}

1. Meyts I, Bucciol G, Quinti I, Neven B, Fischer A, Seoane E, et al. Coronavirus disease 2019 in patients with inborn errors of immunity: an international study. J Allergy Clin Immunol. 2020;147(2):520-31.

2. Bastard P, Rosen LB, Zhang Q, Michailidis E, Hoffmann HH, Zhang Y, et al. Autoantibodies against type I IFNs in patients with life-threatening COVID-19. Science. 2020;370(6515).

3. Toubiana J, Okada S, Hiller J, Oleastro M, Lagos Gomez M, Aldave Becerra JC, et al. Heterozygous STAT1 gain-of-function mutations underlie an unexpectedly broad clinical phenotype. Blood. 2016;127(25):3154-64.

4. van de Veerdonk FL, Plantinga TS, Hoischen A, Smeekens SP, Joosten LA, Gilissen C, et al. STAT1 mutations in autosomal dominant chronic mucocutaneous candidiasis. N Engl J Med. 2011;365(1):54-61.

5. Forbes LR, Vogel TP, Cooper MA, Castro-Wagner J, Schussler E, Weinacht KG, et al. Jakinibs for the treatment of immune dysregulation in patients with gain-of-function signal transducer and activator of transcription 1 (STAT1) or STAT3 mutations. J Allergy Clin Immunol. 2018;142(5):1665-9.

6. Cao Y, Wei J, Zou L, Jiang T, Wang G, Chen L, et al. Ruxolitinib in treatment of severe coronavirus disease 2019 (COVID-19): a multicenter, single-blind, randomized controlled trial. The Journal of allergy and clinical immunology. 2020;146(1):137-46 e3.

7. Zimmerman O, Rosler B, Zerbe CS, Rosen LB, Hsu AP, Uzel G, et al. Risks of ruxolitinib in STAT1 gain-of-function-associated severe fungal disease. Open Forum Infect Dis. 2017;4(4):ofx202.

8. Aydillo T, Gonzalez-Reiche AS, Aslam S, van de Guchte A, Khan Z, Obla A, et al. Shedding of Viable SARS-CoV-2 after immunosuppressive therapy for cancer. N Engl J Med. 2020;383(26):2586-8. 
9. Yan B, Freiwald T, Chauss D, Wang L, West E, Mirabelli C, et al. SARS-CoV-2 drives JAK1 2 dependent local complement hyperactivation. Science Immunology. 2021;6(58):eabg0833.

10. Koschmieder S, Jost E, Cornelissen C, Muller T, Schulze-Hagen M, Bickenbach J, et al. Favorable COVID-19 course despite significant comorbidities in a ruxolitinib-treated patient with primary myelofibrosis. Eur J Haematol. 2020;105(5):655-8.

11. Winthrop KL, Brunton AE, Beekmann S, Polgreen P, Baddley J, Saag KG, et al. SARS CoV-2 infection among patients using immunomodulatory therapies. Ann Rheum Dis. 2021;80(2):269-71.
12. Kalil AC, Patterson TF, Mehta AK, Tomashek KM, Wolfe CR, Ghazaryan V, et al. Baricitinib plus Remdesivir for Hospitalized Adults with Covid-19. N Engl J Med. 2021;384(9):795-807.

Publisher's note Springer Nature remains neutral with regard to jurisdictional claims in published maps and institutional affiliations. 